\title{
Long Survival of Primary Pineal Melanoma with Radiation Treatment Only
}

\author{
Jane Barron, Carolyn Morris-Larkin, Terry Finch, Falah Maroun, Nanette Hache, \\ George M. Yousef
}

Can. J. Neurol. Sci. 2007; 34: 251-253

Pineal gland tumors in adults account for about 0.4 to $1 \%$ of all intracranial tumors. ${ }^{1}$ A wide variety of tumors can occur in the pineal gland. The most common are the pineal parenchymal tumors (pineocytomas, pineoblastomas, and pineal parenchymal tumors of intermediate differentiation), germ cell tumors, and glial tumors. Uncommonly in the pineal gland one may encounter a metastatic tumor, a meningioma or a melanoma. ${ }^{2}$

We here report a case of primary pineal gland melanoma with prolonged survival of 56 weeks after radiotherapy alone. To our knowledge, this is the first reported case of prolonged survival after radiotherapy alone, without surgical intervention or chemotherapy. Contrary to previous reports, ${ }^{3}$ our case demonstrates that a pineal melanoma with leptomeningeal dissemination can still yield a better survival with radiation treatment only.

A 73-year-old woman presented with an episodic history of headache, gait unsteadiness, double vision, and memory change. On neurological examination, she had double vision on extreme lateral gaze and limitation of upward gaze. There was a positive Romberg. Computerized tomography showed a 23 X $17 \mathrm{~mm}$ heterogeneous, predominantly high signal intensity, mass lesion in the pineal region and involving the tectum. Magnetic resonance $\mathrm{T} 1$ weighed images demonstrated the mass to have high signal intensity and very little enhancement (Figure 1). The mass was isointense to white matter on the T2 weighed images and there was increased signal on the proton density scans. Radiotherapy of the pineal area commenced with a radical course of radiation for 6,000 cGy's in 30 fractions using $18 \mathrm{MV}$ photons in a period of 45 days uneventfully. Upon completion of the radiotherapy a repeat MRI showed no significant change in the mass. The patient's condition remained stable for 16 months. She then started to experience some decline in function with periods of confusion, urinary and fecal incontinence, and gait unsteadiness. Seventeen months after her initial presentation, she presented to the emergency department with a two-day history of fever, chills, and a progressive decline in her level of consciousness. Neurological examination then revealed mild hyperreflexia of the lower extremities. The CT scan and MRI demonstrated the pineal tumor but now multiple nodules on the leptomeninges were seen.

Seven days later she developed deep vein thrombosis and was treated with intravenous Heparin. On the fourth day of treatment, she became acutely short of breath and died. Autopsy findings confirmed the presence of pulmonary emboli secondary to deep venous thrombosis.

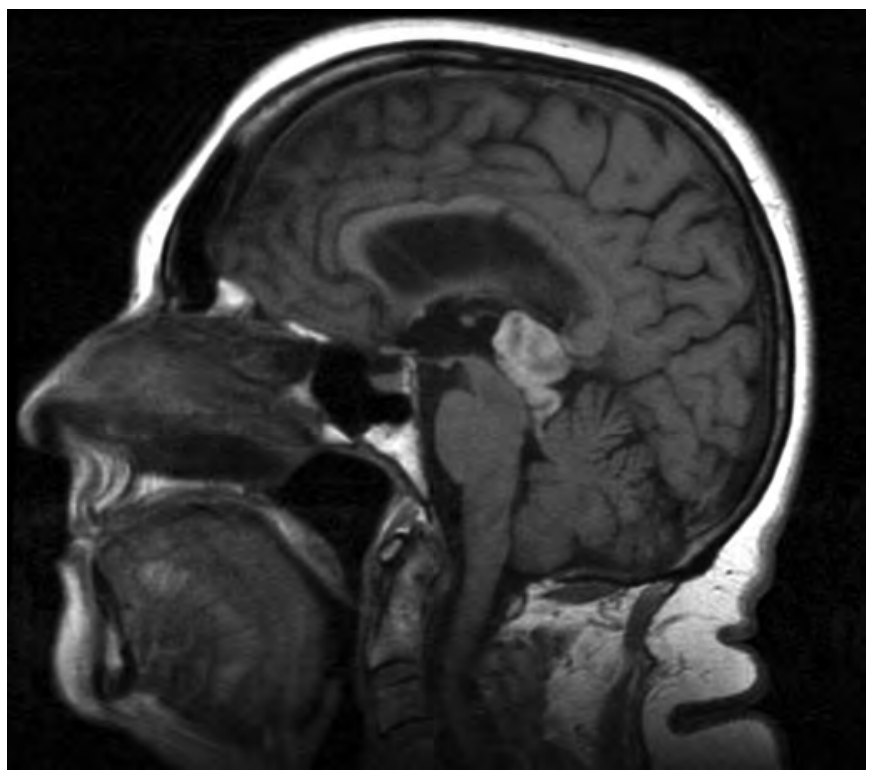

Figure 1. MR T1 weighted image showing a $23 \times 17 \mathrm{~mm}$ high signal pineal region tumor involving the tectum.

\section{Pathological Findings}

A complete autopsy was performed. Examination of the brain showed a soft black pigmented nodule with irregular margins measuring $3 \times 2 \times 1.5 \mathrm{~cm}$ in the area of the pineal gland (Figure 2). In addition, there was multiple black brown nodules $0.5 \mathrm{~cm}$ in average diameter located in the grey and white matter of the cerebrum and cerebellum. Leptomeningeal involvement was also visualized. Thorough ante-mortem investigations and autopsy examination revealed no other primary lesion in other

From the Discipline of Pathology (JB, CML, TF, GMY), Department of Neurosurgery (FM), Department of Radiology (NH), Health Sciences Center, St. John's, NL, Canada. ReCEIVEd July 12, 2006. ACCEPTED In FinAl FORM JANUARY 13, 2007.

Reprint requests to: George Yousef, Health Sciences Center, 300 Prince Philip Drive, St. John's Newfoundland, A1B 3V6, Canada. 


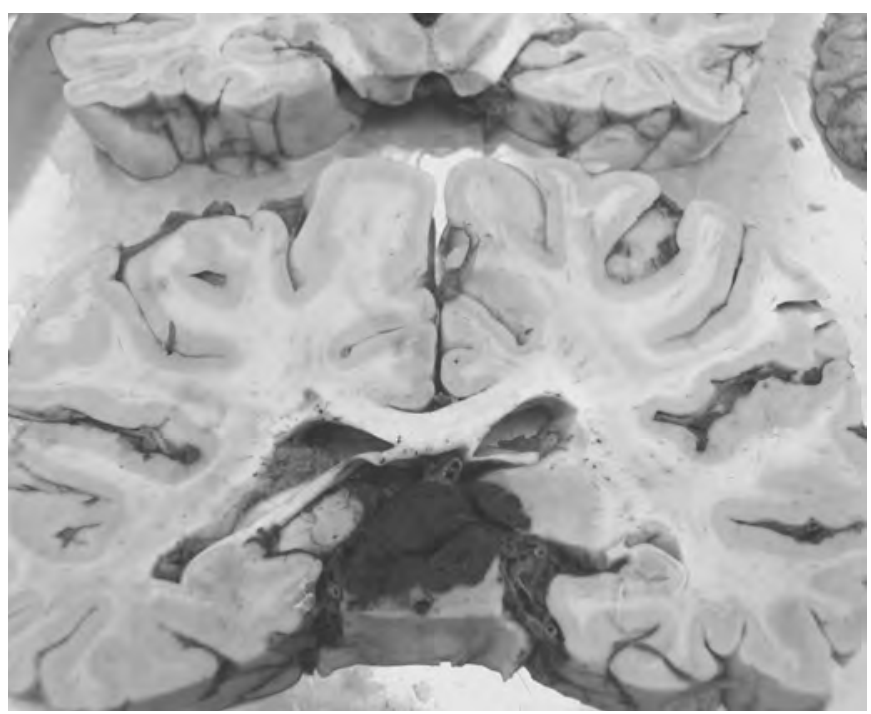

Figure 2. Gross photograph showing total replacement of the pineal gland by tumor with infiltration to the brain stem and thalamus.

organs or tissues. Pigmented skin lesions were seen, but all showed features of benign nevi.

Microscopically, there was almost complete replacement of the native pineal gland by the tumor mass with only the microcalcifications of the native gland identifiable (Figure 3). The tumor was formed of atypical spindled and epitheloid cells (Figure 4) with prominent nucleoli and variable cytoplasmic melanization. Cells showed significant atypia and pleomorphism. Mitotic rate was 1-2/10 hpf (which might be an underestimation due to delayed fixation of the autopsy material). Necrosis was focally present. Scattered tumor cells were also

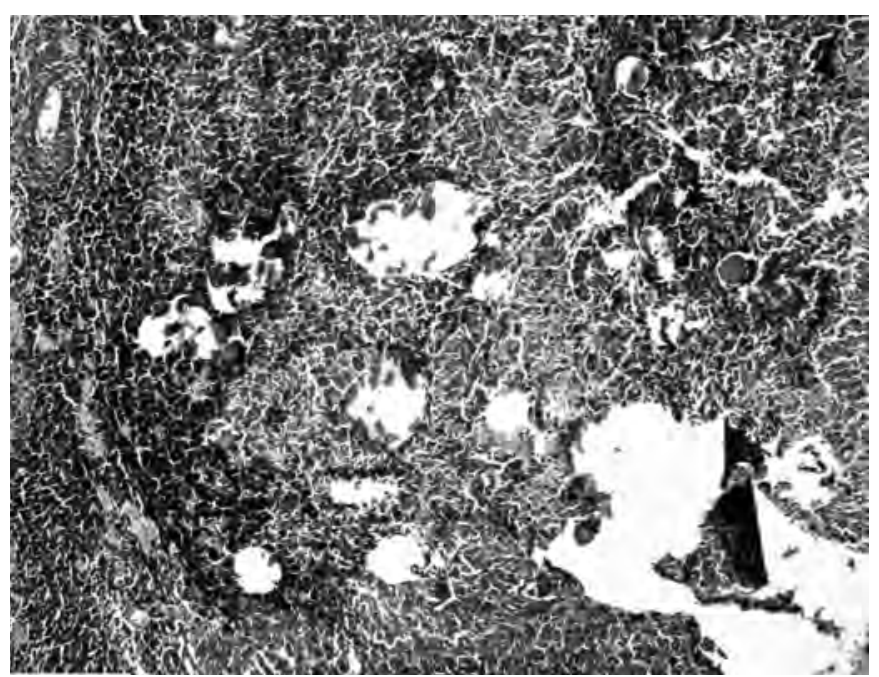

Figure 3. Photomicrograph showing heavily pigmented tumor replacing the pineal gland. Residual calcification of the pineal gland can be seen ( $H \&$ E stain, original magnification X40).

seen infiltrating into the grey and white matter. Immunohistochemical staining to S100, HMB45, and melan A (after bleaching) confirmed the nature of the tumor to be a malignant melanoma (Figure 5). This was also confirmed by electron microscopy. All metastatic nodules showed similar histology.

\section{Discussion}

A primary melanocytic tumor in the pineal region is a rare finding with only 11 cases reported in the literature. The prognosis is grim with a mean survival rate ranging from 4 to 56
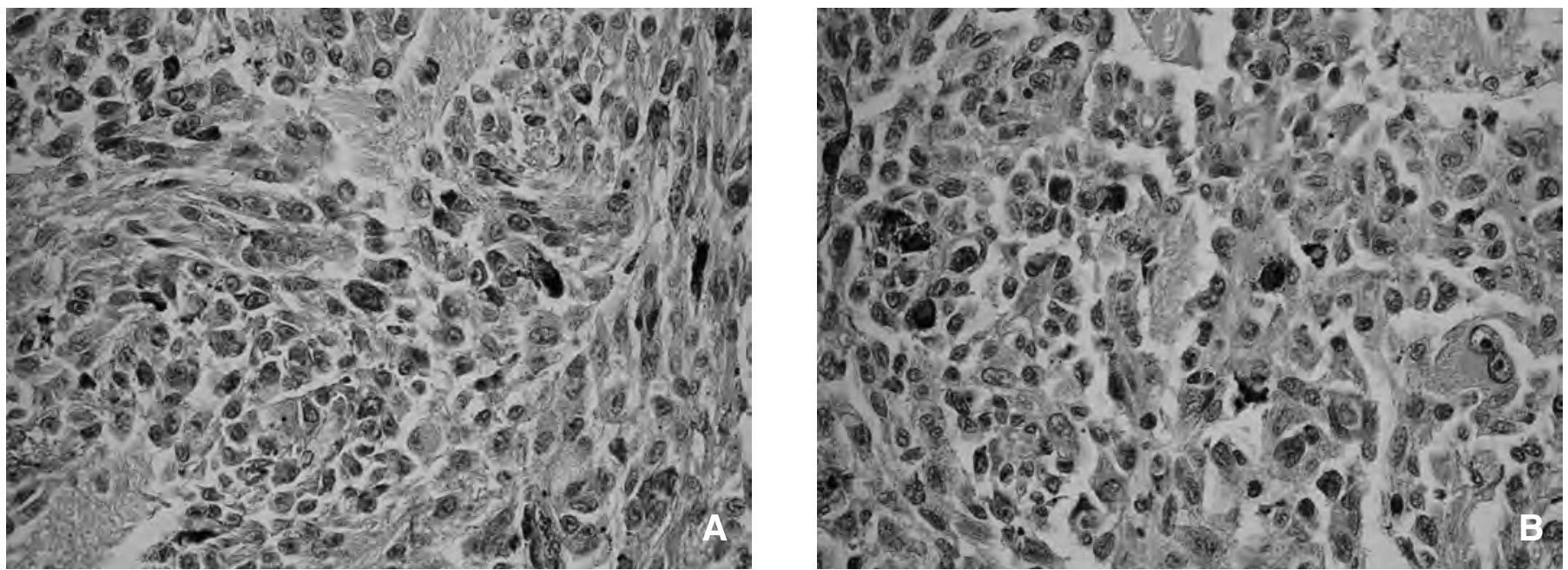

Figure 4. High power magnifications of the tumor, formed of atypical spindled and epitheloid cells with prominent nucleoli and variable cytoplasmic melanization. Cells showed significant atypia and pleomorphism. ( $H \&$ \& stain, original magnification X400). 

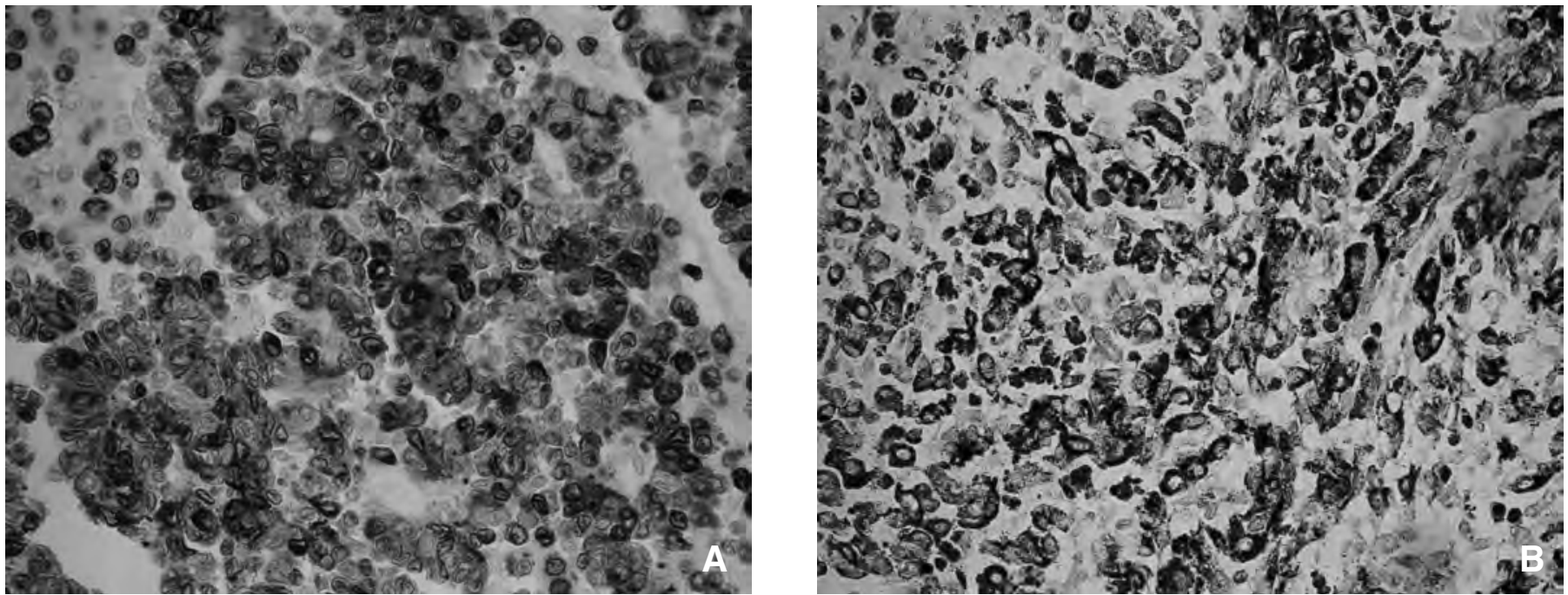

Figure 5. Immunohistochemical staining for melan $A(A)$ and $H M B 45(B)$, after bleaching, confirmed the nature of the tumor to be a malignant melanoma.

weeks. ${ }^{4}$ Typically, pineal melanomas present with obstructive hydrocephalus, symptoms of superior tectal compression including upward gaze paralysis, and cerebellar ataxia from midbrain compression.

Although the optimal management of patients with primary pineal melanoma remains undetermined, treatment appears to increase the length of survival. A variety of treatment modalities have been used for primary pineal melanomas, including chemotherapy, radiotherapy and surgical resection. ${ }^{4,5}$ Survival of cases with no treatment ranged from 0 - 12 weeks. ${ }^{5}$ Radiotherapy alone was used for one case with 16 weeks survival. ${ }^{6}$ An average of one year survival was obtained after subtotal tumor removal and total brain irradiation ${ }^{4}$ and up to four years after partial removal and adjuvant chemotherapy. ${ }^{3}$ Our case is the first to show a longer survival of approximately 68 weeks after receiving radiotherapy alone without surgical resection or chemotherapy. Contrary to a previous observation, ${ }^{3}$ our case demonstrates that a pineal melanoma with leptomeningeal dissemination can still benefit from radiation treatment alone and yield a better survival.

We believe that our patient's melanoma is primary. Thorough examination of all possible primary sites did not reveal any other melanoma. In addition, the main tumor mass was in the anatomical location of the pineal gland with calcific remnants of the original gland seen at the margins. Chronologically, the pineal lesion was the first to be detected radiologically in his brain.

Primary CNS melanocytic lesions can be divided into benign (melanocytomas) and malignant (melanomas) lesions. The origin of these intramedullary primary melanotic pineal tumors is largely unknown but proposed to arise from displaced perivascular melanoblasts. ${ }^{4}$ A transgenic mouse made with the SV40 early region oncogenic sequences under the influence of the tyrosinase promoter were observed to develop tumors of the pineal region. ${ }^{7}$
Since most melanocytic lesions of the CNS are metastatic, a metastatic tumor must be ruled out before a diagnosis of a primary pineal melanocytic tumor can be given, including histological examination of pigmented skin lesions.

Brat et al, ${ }^{8}$ classified CNS melanocytic tumors according to histologic features into three groups with distinct prognosis. Our tumor clearly fits in the category of "malignant melanoma" which carries the worst prognosis, thus the prolonged survival can not be simply attributed to a benign histology.

In conclusion, we report a rare case of primary melanoma of the pineal gland with prolonged survival of 56 weeks after radiotherapy alone, without surgical intervention or chemotherapy. To our knowledge, this is the first reported case with a prolonged survival after radiotherapy alone.

\section{REFERENCES}

1. Czirjak S, Vitanovic D, Slowik F, Magyar A. Primary meningeal melanocytoma of the pineal region. Case report. J Neurosurg. 2000; 92:461-5.

2. Hirato J, Nakazato Y. Pathology of pineal region tumors. J Neurooncol. 2001; 54:239-49.

3. Yamane K, Shima T, Okada Y, Nishida M, Okita S, Hatayama T, et al. Primary pineal melanoma with long-term survival: case report. Surg Neurol. 1994; 42:433-7.

4. Rubino GJ, King WA, Quinn B, Marroquin CE, Verity MA. Primary pineal melanoma: case report. Neurosurgery. 1993; 33:511-5; discussion 515 .

5. Suzuki T, Yasumoto Y, Kumami K, et al. Primary pineal melanocytic tumor. Case report. J Neurosurg. 2001; 94:523-7.

6. Brain tumor registry of Japan. Neurol Med Chir (Tokyo) 1992; 32:381-547.

7. Taylor MD, Mainprize TG, Squire JA, Rutka JT. Molecular genetics of pineal region neoplasms. J Neurooncol. 2001; 54:219-38.

8. Brat DJ, Giannini C, Scheithauer BW, Burger PC. Primary melanocytic neoplasms of the central nervous systems. Am J Surg Pathol. 1999; 23:745-54. 